\title{
Enjeux de l'irrigation pour l'agriculture et disponibilité de la ressource en eau
}

\author{
Gilbert Bros \\ Président du Réseau national d'expérimentation et \\ de démonstration d'hydraulique agricole
}

Dans un contexte global de surproduction entraînant une redéfinition de la PAC (politique agricole commune) avec diminution des prix et mise en jachère d'une partie de la SAU (surface agricole utilisable), l'irrigation peut apparaitre comme une aberration. Aberration d'autant plus grande que plusieurs années de sécheresse estivale sévère ont entraîné dans différentes régions des difficultés pour assurer à la fois, l'alimentation des installations d'irrigation, l'approvisionnement en eau potable, l'alimentation des industries, la préservation d'un débit d'étiage suffisant dans les rivières, et le fonctionnement correct des centrales électriques et des stations d'épuration.

L'irrigation fait pourtant partie intégrante de l'itinéraire technique de production d'un certain nombre de cultures si l'agriculteur veut éviter le risque de ne pas couvrir ses charges et s'il veut satisfaire aux exigences de qualité de ses acheteurs.

L'irrigation est une nécessité, son développement dans les régions de plus en plus septentrionales en est la preuve. Mais elle consomme l'eau à une période où, par définition, l'alimentation naturelle fait défaut. Une politique d'aménagement de la ressource est indispensable pour donner satisfaction à tous les consommateurs potentiels.

\section{L'irrigation, une nécessité pour favoriser la qualité}

Une agriculture moderne compétitive suppose une maîtrise de la production pour assurer et conserver ses débouchés intérieurs et extérieurs. Cette maîtrise passe souvent par celle de l'eau au champ et notamment par l'irrigation.

Les avantages de l'irrigation ne sont plus à démontrer :

- elle permet une croissance beaucoup plus régulière des plantes qui ne souffrent pas d'à-coups de végétation ;

- elle est un moyen de production permettant d'assurer le revenu tout en donnant accès à la possibilité de se diversifier, de modifier l'assolement par l'introduction de cultures à revenu plus élevé dont la réussite est conditionnée par une alimentation en eau régulière et suffisante; - elle assure la qualité des produits, notamment en cultures légumières, et en arboriculture.
Après s'être développée dans les zones de prédilection du maïs, chaudes mais aussi sèches en été, elle a gagné le Centre-Ouest puis des zones plus septentrionales où elle permet des cultures de légumes, de pommes de terre, dans des conditions permettant de répondre aux exigences de quantité et de qualité des industriels. Dans ces régions (Bretagne, Picardie, Champagne-Ardennes notamment) il est difficile voire impossible d'obtenir un contrat de production sans disposer d'une installation d'irrigation. Dans de nombreux secteurs, l'irrigation, moyen indispensable pour maintenir l'activité agricole, est un véritable outil d'aménagement du territoire. L'irrigation a ses avantages, elle a aussi ses contraintes, d'une part elle nécessite de mobiliser en été de l'eau en quantité suffisante à un prix modeste. Ce n'est pas possible partout et cela limite les possibilités de diversification d'un certain nombre d'agriculteurs.

D'autre part, l'irrigation entraîne des investissements importants qui ne peuvent pas être engagés à la légère. Des investissements globaux de 20 à $30000 \mathrm{~F}$ par hectare induisent des coûts d'irrigation supérieurs à $2000 \mathrm{~F} / \mathrm{ha}$ (pouvant aller jusqu'à $3500-4000 \mathrm{~F}$ et plus). Les nombreux investissements réalisés sans aide, ni de l'Etat ni des collectivités, prouvent l'intérêt des agriculteurs pour cette technique et sa nécessité économique.

Les productions des surfaces irriguées sont quelque peu remises en cause. Non seulement le revenu hectare va baisser du fait de la diminution des prix, mais les charges vont aussi augmenter; en effet, l'absence de diversification possible chez un certain nombre d'irrigants, le gel des terres, les obligent à amortir leurs investissements sur des surfaces plus faibles. Si la mobilisation de la ressource en eau a exigé des investissements lourds, dans le cadre d'ASA ou d'ASL par exemple, le coût du $\mathrm{m}^{3}$ utilisé risque d'augmenter dans des proportions telles qu'il serait insupportable et cela peut remettre en cause l'existence même de ces associations. Les nombreuses simulations réalisées actuellement afin de mieux cerner le revenu futur des agriculteurs doivent impérativement prendre en compte ces phénomènes sans oublier les problèmpes que pose la gestion quotidienne d'une installation d'irrigation qui n'est plus vraiment adaptée. 


\section{En vingt ans, la surface irriguée a largement doublé}

Sur quelque 30 millions d'ha SAU, 1,2 Ms sont irrigués annuellement pour une surface équipée d'1,8 Ms ; 120000 exploitations sont concernées. En 1975, 508000 ha seulement étaient irrigués. Depuis une dizaine d'années, l'effort d'équipement porte sur 40 à 50000 ha/an.

Le maïs représente à lui seul la moitié de la surface irriguée. Culture exigeante en chaleur et en eau, elle ne tolère pas le déficit hydrique en juillet-août. Le maïs permet de bien valoriser l'eau. Au départ, localisée dans la Beauce et au Sud-Ouest, son irrigation s'est largement développée en Poitou-Charentes, Pays de Loire, Alsace notamment.

A côté des surfaces irriguées en fourrages et plantes sarclées qui ont légèrement diminué, de nouvelles cultures comme le tournesol et surtout le soja n'ont pu se développer que grâce à l'irrigation. En 12 ans, quelque cent mille hectares ont été équipés pour irriguer ces cultures.

Quant aux légumes frais et de conserve, à la pomme de terre, leur surface irriguée est en légère augmentation. On constate que ces productions se développent notamment en, Nord-Picardie, Champagne-Ardennes et même Bretagne, régions considérées communément comme humides. Ce développement se fait souvent sous la pression des industriels qui recherchent des rendements et une qualité aussi constants que possible.

Deux grandes catégories de cultures sont donc irriguées :

- des grandes cultures : maïs, soja, tournesol, mais aussi pois, blé, fourrages dont la rentabilité est de plus en plus difficile à assurer compte tenu du contexte ;

- des cultures à haut produit net: légumes, semences, arboriculture, pour lesquelles l'irrigation ne représente qu'une faible partie du coût de production. Ces cultures sont un moyen de se diversifier. Leurs débouchés ne sont pas élastiques. L'irrigation peut aussi assurer la protection antigel et permettre de régulariser l'approvisionnement du marché, avec un produit de qualité constante.

\section{L'irrigation implique une mobilisation de la ressource en eau}

A côté de régions comme la Beauce ou le Sud-Ouest où l'irrigation s'est implantée puis développée sur une bonne trentaine d'années, d'autres régions ont vu leur surface irriguée multipliée par 2 ou 3 parfois plus en moins de 10 ans. Ce développement entraîne des prélèvements élevés en été dans un milieu qui ne dispose pas toujours de ressources faciles à mobiliser à grande échelle.

Globalement, la France ne manque pas d'eau. Sur les $400 \mathrm{Mds}$ de $\mathrm{m}^{3}$ de précipitations qui tombent tous les ans sur le territoire, on admet que le quart, soit $100 \mathrm{Mds}$ de $\mathrm{m}^{3}$ est exploitable. Les prélèvements représentent actuellement $41 \mathrm{Mds}$ de $\mathrm{m}^{3}$, dont plus de la moitié pour les centrales électriques, $15 \%$ pour l'eau potable, $12 \%$ pour les industries non raccordées à un réseau, $11 \%$ pour l'agriculture. Mais prélèvement n'est pas synonyme de consommation.
En fait, sur les 41 Mds prélevés, 8 Mds seulement sont consommés, c'est-à-dire non réinjectés dans le réseau des rivières après traitement ou utilisation. Sur ces $8 \mathrm{Mds}$ de $\mathrm{m}^{3}, 3$ sont consommés par l'agriculture, essentiellement pour l'irrigation. En été, l'agriculteur irrigant est le plus gros consommateur d'eau ( 60 à $80 \%$ selon les régions). A l'hectare irrigué, la consommation représente, en moyenne, $2500 \mathrm{~m}^{3}$. Ces dernières années, la consommation d'eau par irrigation a augmenté de $10 \%$ tous les 3 ans du fait de l'accroissement des surfaces irriguées. L'accroissement global de la consommation d'eau a été cependant limitée par un ensemble d'actions visant à mieux conduire l'irrigation en fonction des besoins des plantes et des réserves en eau du sol, mais aussi à utiliser le matériel de façon plus rationnelle (voir les communications de MM. Rieul et Dubalen).

Les opérations de contrôle des matériels d'irrigation au champ menées d'abord par le Bcma puis par les conseillers irrigation de plusieurs régions avec le concours du RNEDHA permettent d'inciter les irrigants à mieux maîtriser les réglages de leur matériel. De leur côté, les constructeurs ont mis sur le marché des enrouleurs sur lesquels la régulation de la vitesse d'avancement est plus fiable grâce à l'adoption de l'électronique.

Les constats effectués sur le terrain montrent que des progrès importants sont encore possibles pour économiser l'eau. Des actions sont en cours dans différentes régions pour démultiplier les résultats des opérations diagnostic.

Le développement rapide de l'irrigation entraîne donc une consommation importante en période estivale dans un milieu qui ne dispose pas de ressources suffisantes pour soutenir les étiages et faire face aux besoins des différents consommateurs d'eau. Un décalage important existe dans plusieurs régions entre le volume d'eau prélevé dans les cours d'eau en période de besoins élevés pour l'agriculture et les réserves de soutien d'étiage disponibles, d'où des conflits entre les différents usagers de l'eau dont les irrigants sont souvent la cible privilégiée. L'eau pourtant existe mais les eaux de surface s'évacuent rapidement et on a tout fait pour qu'il en soit ainsi : drainage, destruction des haies, reprofilage des fossés, des rivières. L'eau ne peut plus s'infiltrer assez longtemps ce qui réduit la migration vers les nappes souterraines et le stockage dans le sol.

Quant aux nappes souterraines, leur volume est élevé, on l'estime à $1000 \mathrm{Mds}$ de $\mathrm{m}^{3}$ mais elles ne sont pas toujours situées dans les régions les plus demandeuses et leur accessibilité est parfois coûteuse.

La sécheresse de ces dernières années a montré dans plusieurs régions des déséquilibres criants entre demande et ressource. Elle a amplifié les conséquences d'une augmentation constante des besoins en eau à tous les niveaux (irrigation, eau potable, industrie, tourisme). Ces difficultés traduisent un décalage entre l'augmentation de la consommation en été et les efforts d'investissements réalisés pour mobiliser une ressource suffisante pour faire face à tous les besoins recensés. La sécheresse a révélé un déficit de ressource structurel et non conjoncturel qui pénalisent les irrigants auxquels on interdit d'utiliser leur installation. Pour remédier à cette situation, des efforts doivent 
être faits pour créer des ressources en eau suffisantes par la construction d'ouvrages de retenue et de régulation des eaux. Ces constructions sont possibles car la ressource existe. En effet, les prélèvements actuels sont faibles par rapport à ce qui s'écoule dans les rivières (moins de $10 \%$ ) et la consommation nette d'eau est insignifiante. Le déséquilibre très important constaté entre demande et disponibilité est trop grand pour qu'il puisse être résorbé par simple action sur la demande, il faut mettre en place, comme le préconise le rapport Mangin, une politique de mobilisation de la ressource combinant les grands bassins, les barrages agricoles, les ouvrages de transfert entre barrages, les retenues collinaires. Une prospection des ressources en eaux souterraines est également indispensable.

La mobilisation de la ressource ne peut plus se faire de façon anarchique. Dans la situation que nous connaissons, l'eau n'est pas un dû. Les utilisateurs doivent respecter la discipline permettant à chacun d'en disposer en quantité suffisante au moment où il en a besoin. La constitution raisonnée de réserves alimentées par les excédents, que l'on contrôle mal, voire pas du tout, éviterait que l'agriculture ne soit systématiquement prise comme bouc émissaire chaque fois que les étés se révèlent un peu secs.

La mobilisation de la ressource doit s'appuyer sur des études sérieuses, établissant avec le maximum de précisions, le potentiel des bassins versants. Face aux potentiels, un calcul des besoins à satisfaire dans les différents domaines doit être effectué avec attention. Pour éviter les investissements excessifs, la détermination des volumes nécessaires doit s'appuyer à la fois sur une prospective réaliste et la mise en place de mesures d'accompagnement incitant aux économies d'eau (par exemple, vérification de l'étanchéité des réseaux, meilleure conduite de l'irrigation).

La loi sur l'eau, du 3 janvier 1992, se propose notamment de mettre en place une politique globale raisonnée par bassin versant pour la constitution de réserve et la mise à disposition des nappes. Elle vise à établir, dans un délai de 5 ans, un ou des schémas directeurs d'aménagement et de gestion des eaux, fixant pour chaque bassin ou groupement de bassins, les orientations fondamentales d'une gestion équilibrée des ressources. Plus localement, des schémas d'aménagement et de gestion des eaux (SAGE) doivent fixer les objectifs généraux d'utilisation, de mise en valeur et de protection des ressources en eaux superficielles et souterraines. Ce schéma sera élaboré et suivi par une commission locale de l'eau.

Cette démarche proposée par la loi paraît nécessaire mais il paraît tout aussi indispensable de mettre en cuvre les moyens financiers suffisants pour la mise en place effective des nouvelles ressources. Dans plusieurs régions, il faudrait déjà dégager, dans les meilleurs délais, les moyens pour rattraper le retard accumulé afin de pouvoir rétablir des débits suffisants en période d'étiage et, dans un second temps, créer des ressources nouvelles pour satisfaire les besoins dans tous les secteurs de l'activité économique.

Dans le contexte économique actuel, les agriculteurs ralentissent leurs investissements, les équipements sont les premiers touchés et la demande pour de nouvelles installations d'irrigation se ralentit, il faut mettre à profit cette période pour mettre en place les ressources dont tous les utilisateurs ont besoin. 